\title{
Rheological Assessment - A Road Map for Plant Designers and Operators
}

\author{
F. Sofra Rheological Consulting Services Pty Ltd, Australia
}

\section{INTRODUCTION}

One of the fundamental prerequisites for the design and implementation of a successful thickened tailings or paste systems is a comprehensive understanding of the rheological characteristics of the material. Although rheological characterisation is often out-sourced, it is essential that design engineers and plant operators have a grasp of the variables which must be considered, the questions that need to be addressed and familiarity with rheological assessment principles in order to determine the validity of data provided for the purpose intended. Good rheological data for one particular sample under one specific set of testing conditions is generally insufficient for design and/or optimisation of entire processing systems and the related equipment. An 'up-front' understanding of how rheological parameters affect and influence operations will result in more efficient and sustainable plant performance and can mitigate many potential problematic issues. This paper is designed to be a rheology road map, guiding the reader through a checklist of rheological questions to ask and issues to address - from sampling to testing protocols, data analysis and informed data application.

\section{OUTLINE}

This paper aims to highlight, and demonstrate by example some of the critical issues for effective rheological assessment. These issues include the practice of understanding the influence of ore type and processing conditions in the concentrating plant, quantification of the physical characteristics of the feed stream to the tailings plant, measurement of rheological parameters, applicability of the results and techniques to assess these parameters, including testing and modelling.

Not all pertinent issues will be discussed in detail in this limited paper. The intention is to publish a more comprehensive road map within the next year.

\section{ORE VARIABILITY}

The mineralogical composition and physical constitution of ores varies between mineral types and deposits, as well as within a given deposit which will be manifest during the period of extraction. Understanding how these variations impact on the 'processability' of ores and the tailings' flow behaviour is an essential step in quantifying the variability in rheological parameters and thereby mitigating project risk, and/or ensuring sustainable operation of the processing plant. 


\subsection{Mineralogy and particle size distribution}

Where it is known that the ore body contains significant variations in mineralogy, for example clay seams in a coal deposit or limonites and saprolites in a nickel laterite deposit, reconciling mineralogy with the resultant rheological characteristics provides important information for determination of ore blending plans and the expected range of operating conditions for the concentrating plant.

Due to changes in mineralogy and effects such as weathering, there can be large variations in the friability and therefore particle size distribution (PSD) throughout the ore body and during the life of mine. The deviation in PSD must be determined from the outset as particle size dramatically affects the rheology of slurries and pastes and thus the ease of processing and disposal.

Changes in mineralogy and/or PSD within a deposit can mean an order of magnitude variation in the tailings yield stress (the stress required to initiate flow) at a given solids concentration and can be the difference between a successful and unsuccessful tailings disposal operation. Figure 1 illustrates the variations in the yield stress profiles of samples from one lateritic nickel deposit as a result of changes in mineralogy. Note that for a yield stress of $200 \mathrm{~Pa}$, the solids concentration varies from approximately $42 \mathrm{wt} \%$ to $56 \mathrm{wt} \%$, or alternatively, at a solids concentration of $50 \mathrm{wt} \%$, the material properties range from those of a solid i.e. not pumpable with an unmeasurably high yield stress, to a yield stress of about $30 \mathrm{~Pa}$ i.e. easily pumpable with the consistency of cream.

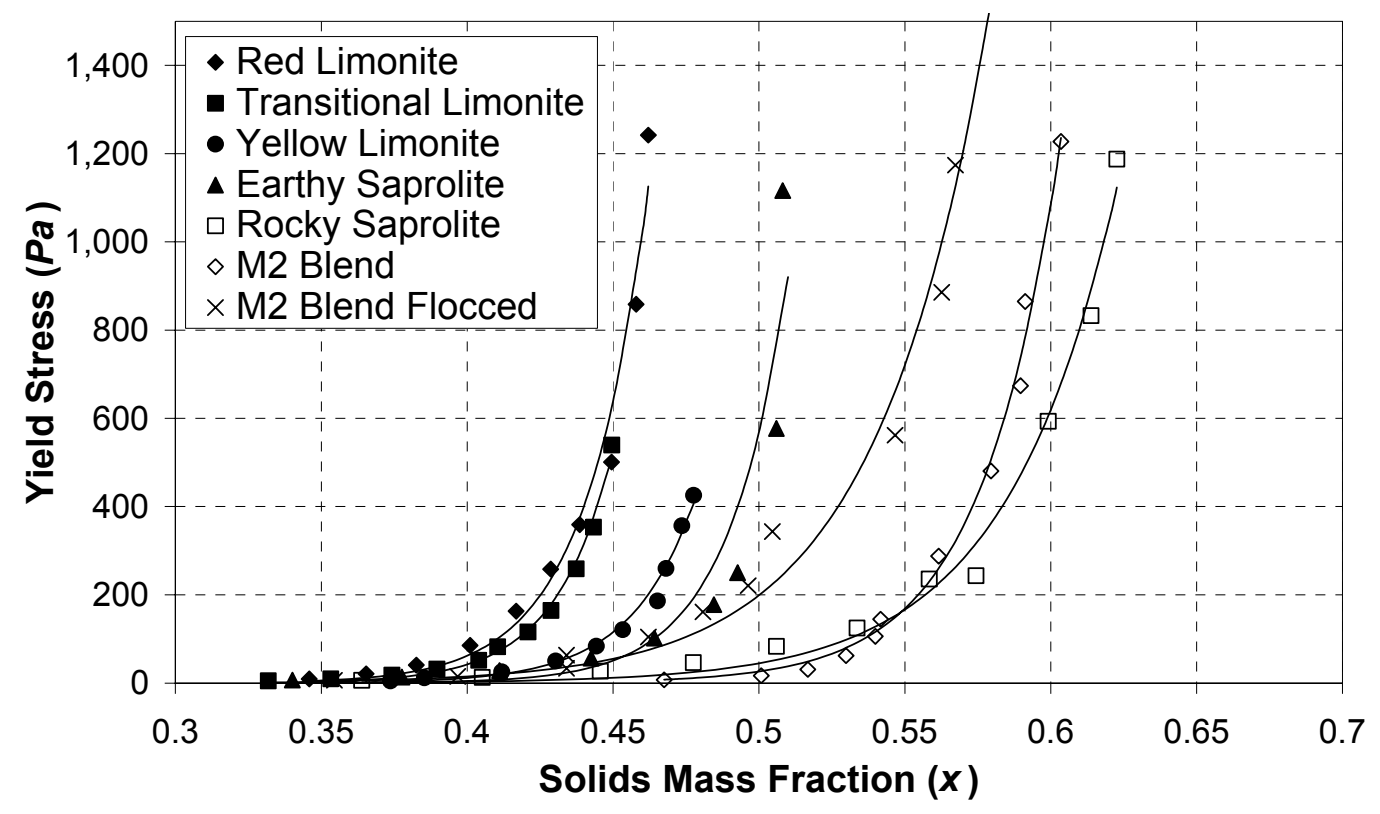

Figure 1 Effect of mineralogy on the yield stress profiles of nickel laterites

An example of the effect of particle size on the yield stress profile of a bauxite sample is shown in Figure 2. As observed for varying mineralogy, changes in the PSD can translate to dramatic changes in the yield stress 
for a given solids concentration. In the case shown, for a solids concentration of $67 \mathrm{wt} \%$, the yield stress could be anywhere from $10 \mathrm{~Pa}$ to $110 \mathrm{~Pa}$.

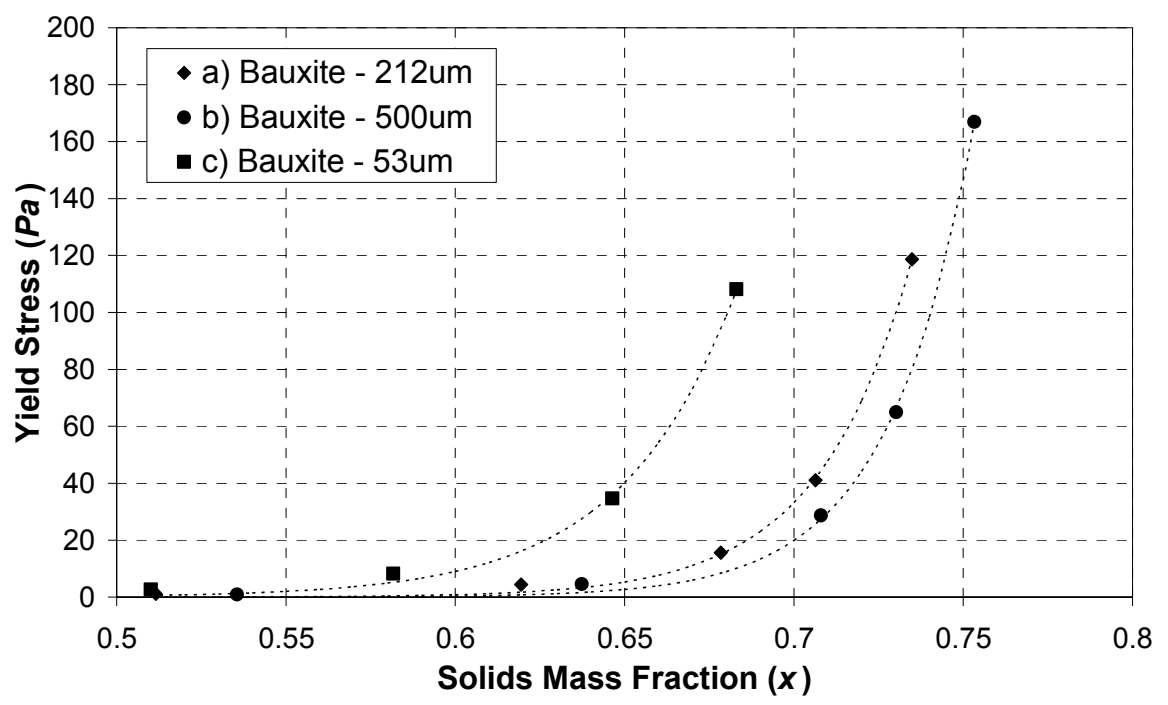

Figure 2 Effect of PSD ( $\left.d_{90}\right)$ on the yield stress profiles of bauxite ores

\subsection{Sample preparation}

The importance of testing a representative sample can not be understated. Those who frequently conduct rheological testwork understand the critical nature of sample preparation and management in order to ensure that the results of their investigations will accurately reflect the applied process conditions.

Whether the samples are a result of pilot plant trials or full scale plant operation, at times there may be a delay between sample generation and testing. In the event of this delay, it is important to consider the effects of sample aging and transport.

Sample aging is particularly important for flocculated samples where the flocculant may degrade over time, samples generated at elevated temperature where cooling may affect the rheology and samples containing clays, where clays may delaminate and swell over time. In these cases, on-site testing is recommended at the time of sample generation and the process temperature.

During transportation to a testing facility both aging and shear history effects may be significant. As the sample is transported it is inevitable that the shear history experienced will differ from plant conditions. Obviously, in the case of shear history dependent materials (where the rheology varies with time of shear, for example flocculated materials, clays, red mud and many nickel tailings) this will result in variations in the measured rheology compared with plant conditions. Settling and particle size segregation is also likely, requiring the sample to be homogenised and resuspended prior to testing.

Often the very act of preparing a sample for testing will alter the rheological properties, especially in the case of flocculated samples where the floc structure may be delicate and easily destroyed. Among the most difficult information to obtain is that required for determination of thickener rake drive torque requirements 
and thickener underflow pump requirements. The material that the rake and underflow pump must deal with will be freshly flocculated and relatively unsheared. However, sample generation, collection and testing will impart shear on the flocculated and thickened sample.

Although extensive efforts are made to minimise the effects mentioned, they are sometimes unavoidable and often the only prudent approach is to ensure the data is utilised with awareness of the limitations imposed by sample preparation.

\section{BASELINE RHEOLOGY TESTWORK}

Although specific information is required for the design of individual unit operations, in general a baseline rheological characterisation consists of the following suite of tests.

\subsection{Particle size distribution (PSD) and mineralogy}

Although not strictly speaking part of rheological testwork, as stated in section 2, reconciliation of the PSD and mineralogy (using x-ray diffraction for example) with the rheological characteristics can be particularly useful for mine planning and blending requirements where there is significant ore variability. Design of operations relies on an understanding from the outset of the variability expected in rheological properties attributable to the ore type, blended or unblended, being processed and transported.

\subsection{Shear history effects}

The simplest method of assessing the effect of shear history is to measure the yield stress (or torque) as a function of shearing time at a fixed shear rate. Many concentrated suspensions, particularly flocculated or coagulated suspensions, posses a networked structure which may be broken down as they are subjected to shear. Figure 3 for example, shows the yield stress reduction for a nickel sample to an equilibrium value with time of shear.

It is important to understand the rate and extent of this breakdown process to ensure rheological testing is conducted at the appropriate shear history condition for which the data is to be applied. In the case of thickener rake drive torque requirements and underflow pump sizing for example, testing should be conducted in the unsheared state as this represents the state of the material that the rake must deal with. For pipeline calculations and prediction of the depositional flow characteristics for slurry/paste that has been sheared by centrifugal pumps, testing may need to be conducted in a fully sheared state to avoid over design of the pumping system. Centrifugal pumps impart a particularly high shear intensity which may reduce the pumping energy requirements through breakdown of the paste structure, thereby reducing the yield stress and viscosity. 


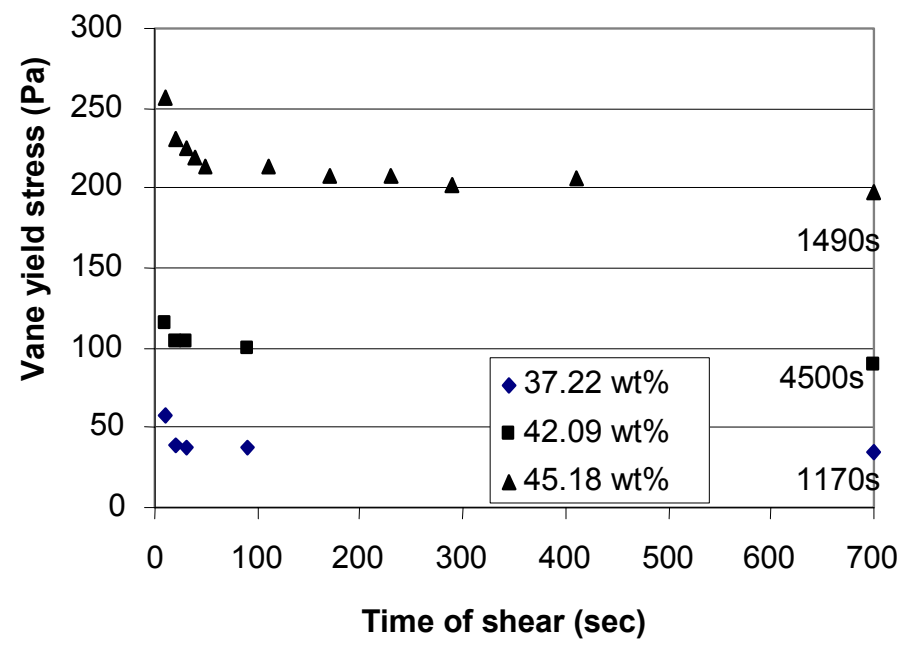

\section{Figure 3 Vane shear yield stress vs time of shear for a nickel sample showing the eventual equilibrium state}

There are times when it is not possible to replicate the shear history conditions anticipated in reality (they are generally not known a priori) so understanding the rate and extent of variation in the rheology is important for informed use of data for design or optimisation.

Although the behaviour shown in Figure 3 is common, there are instances where the effect of shear history varies as a function of the rate of applied shear. The presence of high aspect ratio particles in some tailings results in the observed yield stress either increasing or decreasing depending on the shear history imparted, as shown in Figure 4. The apparent reversibility and range of potential structural states suggests that definition of an equilibrium condition is purely subjective and shear history dependent. In cases where this type of behaviour is evident, it is important to ensure that the shear conditions that will be experienced in the plant are well understood and reproduced as closely as possible during sampling and testing.

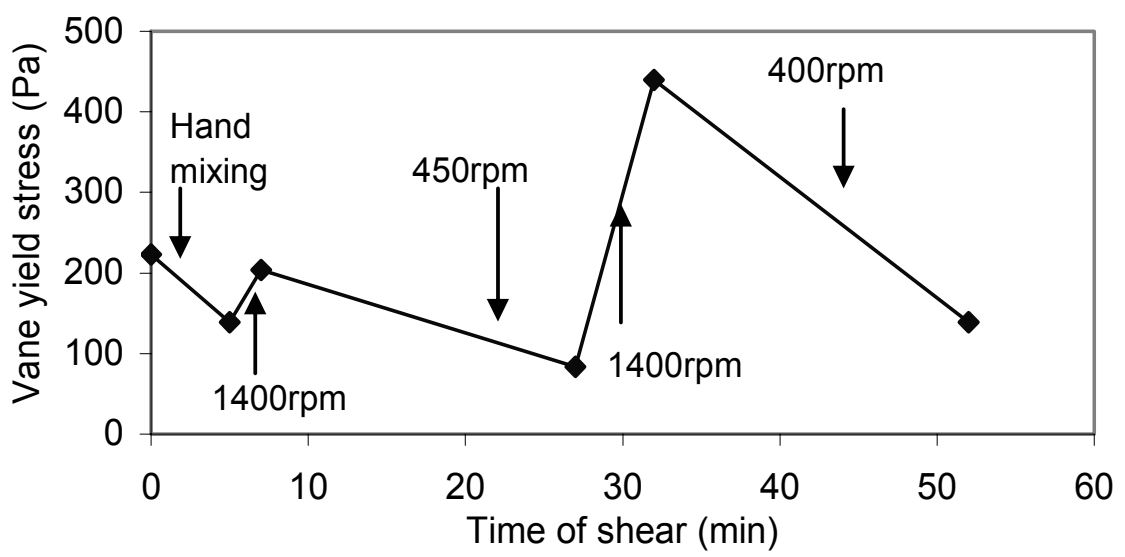

Figure 4 Yield stress progression with varying intensity of applied shear 


\subsection{Yield stress profile}

Following determination of the effect of shear history, the yield stress as a function of solids concentration should be determined for the appropriate shear history condition to define the operating window. Figure 5 illustrates the variation in yield stress behaviour due to shearing for a flocculated thickener underflow tailings sample.

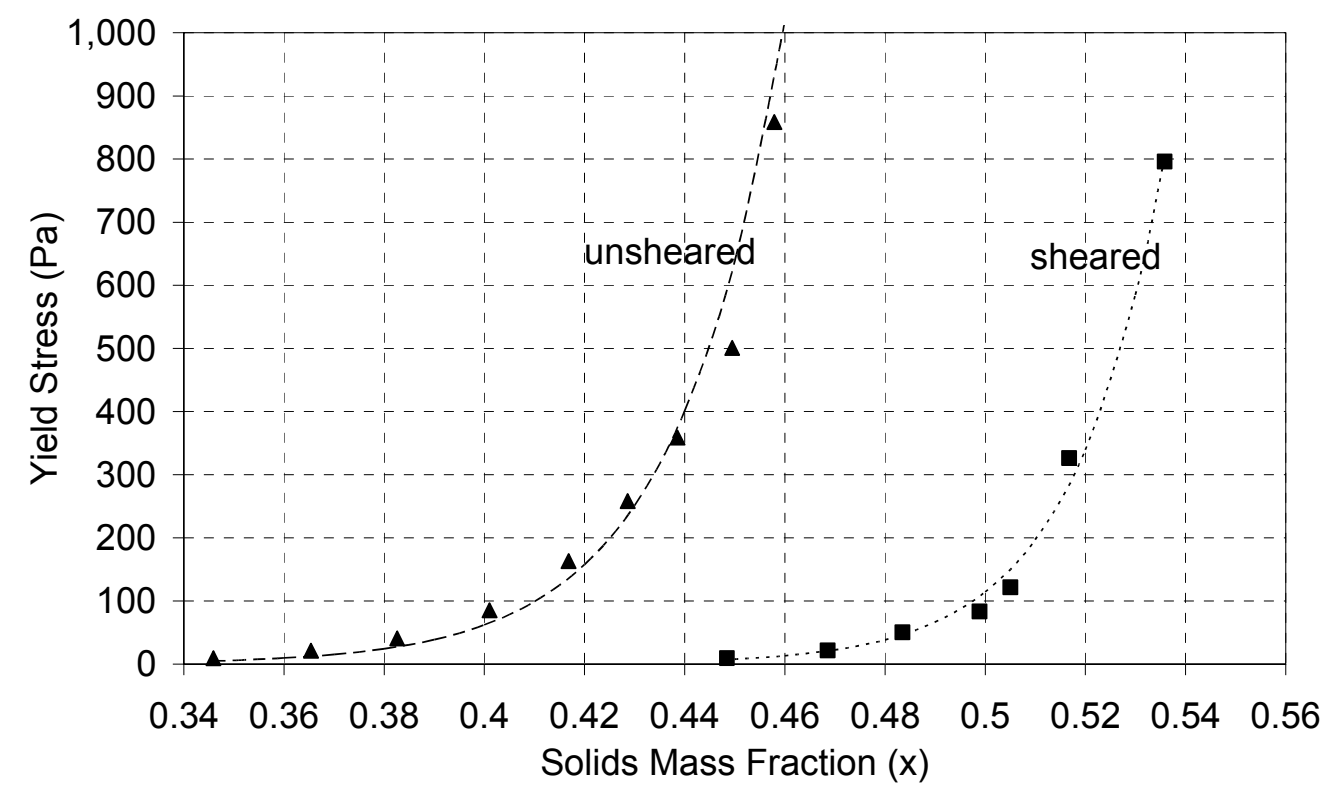

\section{Figure $5 \quad$ Yield stress profiles - unsheared and sheared flocculated thickener underflow}

As shown above, yield stress profiles generally display exponential or power law trends. The yield stress profile should be determined over a wide range of solids concentrations extending beyond the anticipated operating concentration to indicate where on the curve the operational point lies. A design operating point near the 'elbow' of the curve, where the yield stress increases dramatically with minor variations in solids concentration, could be problematic because of the narrow operating window and difficulty controlling the paste concentration to such a specific value.

In addition to the effect of shear, and to further highlight the effect of PSD, a decrease in the PSD will shift the curve to the left i.e. a higher yield stress for a given solids concentration. Conversely, a coarse PSD will shift the curve to the right, but with a more abrupt transition from liquid to solid-like behaviour i.e. a sharper 'elbow' in the curve.

\subsection{Shear stress vs shear rate}

A flow curve, or rheogram measures the shear stress as a function of shear rate and therefore the viscosity as a function of shear rate. Flow curves need to be measured over the anticipated operating range indicated by yield stress profiles and may also need to encompass a range of shear histories (from unsheared to fully sheared). Figure 6 demonstrates the importance of testing under the appropriate conditions by showing the 
effect of shear through the thickener underflow pump and pipeline for a red mud paste. Using the thickener underflow rheology to design the pipeline system or deposition characteristics could lead to serious problems in the process design.

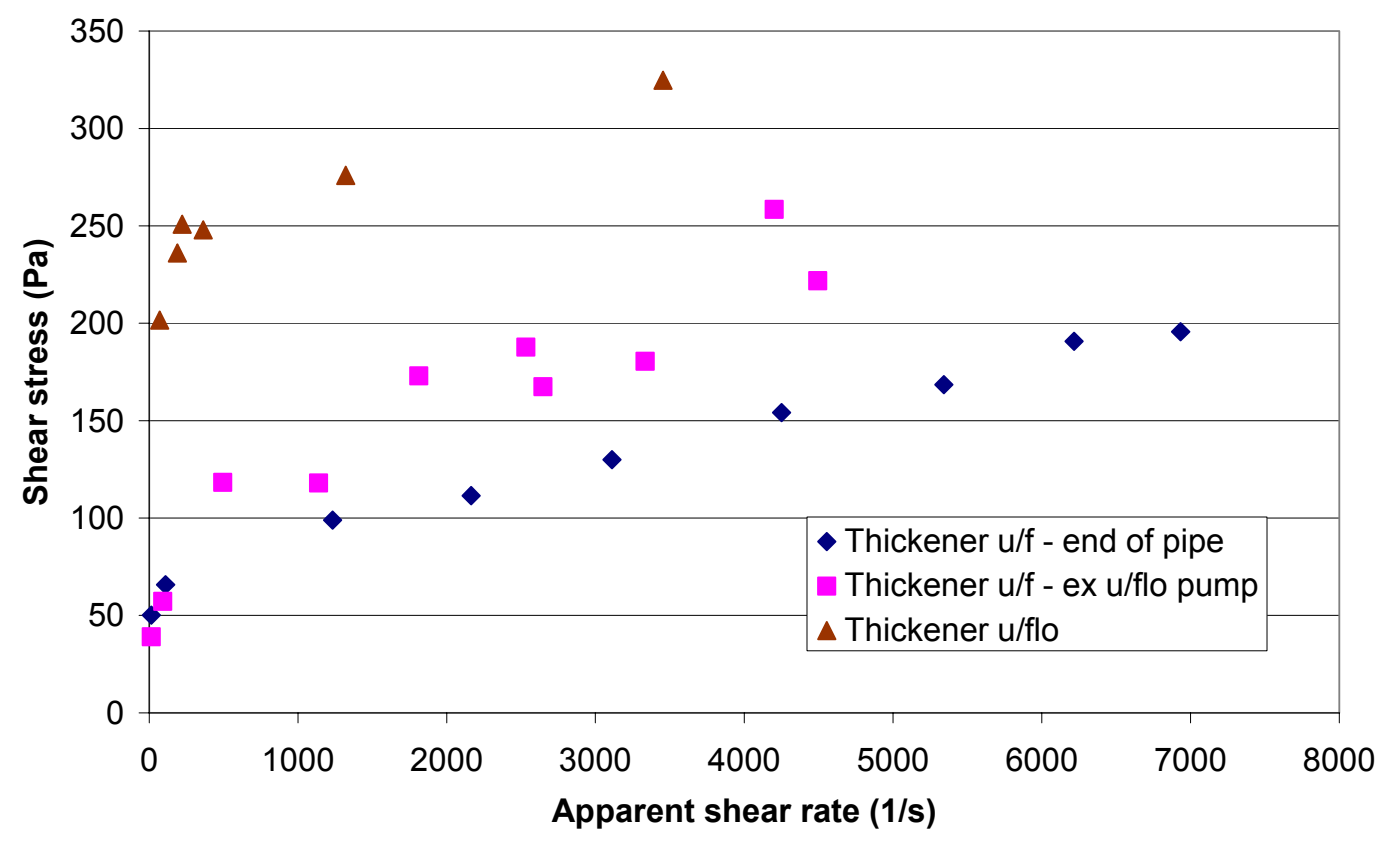

Figure $6 \quad$ Flow curves for a red mud paste showing progressive structural breakdown

\section{TESTING METHODS AND ISSUES}

Some testing methods are more appropriate for a particular application than others. However, what is more important is that those conducting measurements and those applying the data generated have an understanding of the issues associated with various testing methods. Rather than describe particular test methods in detail, this section aims to comparatively discuss critical issues for each method.

\subsection{Slump testing}

Quoting a slump height is useful as a relative measurement for a particular material only. Slump height is not a material property, but rather relates to the yield stress (a material property) through the density. Unless it is used to calculate a yield stress, the slump height is only good for relative measurements for a particular material. Table 1 shows how three samples displaying the same slump height can have very different yield stresses in reality resulting in a wide variation in the pumping energy requirements. Slump testing is most useful as a quick and simple method of comparing sample yield stress or a as an on-site quality control method. 
Table $1 \quad$ Yield stress and pipeline pressure drop for a fixed slump height

\begin{tabular}{|c|c|c|c|c|}
\hline & Coal Tails & Au Tails & $\mathrm{Pb} / \mathrm{Zn}$ Tails & \multirow{6}{*}{$\begin{array}{l}* \mathrm{dP} \text { Prediction assumes } \\
\text { Bingham viscosity }=1 \mathrm{~Pa} . \mathrm{s} \\
\text { Pipe ID }=200 \mathrm{~mm} \\
\text { Pipeline Velocity }=1 \mathrm{~m} / \mathrm{s} \\
\text { Horizontal pipeline }\end{array}$} \\
\hline Solids SG $\left(\mathrm{kg} / \mathrm{m}^{3}\right)$ & 1450 & 2800 & 4100 & \\
\hline Solids conc. $(\% \mathrm{w} / \mathrm{w})$ & 36 & 75 & 75 & \\
\hline Slump height (mm) & $203(8 ")$ & 203 (8”) & $203(8 ”)$ & \\
\hline Yield stress $(\mathrm{Pa})$ & 160 & 275 & 330 & \\
\hline $\begin{array}{l}\text { Predicted pressure drop } \\
(\mathrm{kPa} / \mathrm{m})^{*}\end{array}$ & 5.07 & 8.13 & 9.6 & \\
\hline
\end{tabular}

\subsection{Rotational methods}

Rotational rheology testing, including vane and couette (cup and bob) methods are convenient, being highly portable, requiring small sample volumes and provide a low cost alternative to pipeloop tests (section 5.3). If used prudently rotational methods are accurate, however there are a number of factors to be considered when applying data obtained using rotational devices.

Many testing facilities employ ramped measurements, where the shear stress is measured as the shear rate is progressively increased (or decreased) from one value to the successive value. There are a number of issues associated with this testing approach which need to be considered:

- Each measurement is dependent on the shear rate and duration of shear of preceding measurements in the sweep.

- Particle migration away from the shearing surface may take place over the duration of the test resulting in measurement of a low concentration region not representative of the bulk sample. For smooth bobs rotating in a cup, slip may occur at the bob surface, again resulting in measurement of a low concentration region not representative of the bulk sample.

- For shear thinning or thixotropic slurries, the reduction in the yield stress and viscosity over the duration of the test may result in particle settling and the formation of a concentration gradient along the length of the shearing surface.

- Evaporation at high temperature may be significant, thereby increasing the concentration of the sample being tested over the duration of a test.

For the majority of slurries and pastes, ramped measurements are inappropriate and a superior approach is to conduct individual measurements at successive shear rates with minimal, gentle homogenisation of the sample between measurements.

A good way to check the validity of flow data from rotational testing (or any testing for that matter) is to compare the low shear rate data with an independently measured vane yield stress. If the low shear rate data does not coincide with the yield stress this indicates that one or more of the above factors may be prevalent. 


\section{3 'Pipe' methods}

A tubular geometry is often preferred given the similarity with pipeline flow. Examples include capillary (small tube) rheometry and pipeloop tests. For capillary testing care must be taken to eliminate wall and end effects by ensuring that the tube length and diameter are appropriate for the PSD of the sample. In order to minimise the possibility of wall and end effects, both $L / D$ (tube length / diameter) and $D / d_{50}$ should be greater than about 60 where the $d_{50}$ is the median particle size of the suspended particles.

Pipeloop testing is often performed to verify simpler rheological testing eg. rotational methods, and to indicate the parameters such as settling velocities in the presence of shear. One of the disadvantages with pipeloop tests is that the sample is continually recirculated, making determination of shear history effects difficult. Pipeloop tests are rarely conducted as part of an initial rheological characterisation programme as they are more time consuming, expensive and large sample sizes are required.

\section{DATA ANALYSIS AND APPLICATION}

No matter how 'good' rheological data is, it is imperative that the end-user ensure that rheological information is applied appropriately. One of the first things to consider is whether the data is relative or absolute. Relative data, for example slump heights for a particular material or flow data obtained for a different shear history condition to that of the process, can be useful in it's own right, but the user must be aware that the information may be best for ranking, comparative or 'indication of change' purposes rather than direct design input.

\subsection{Automated instrument analyses}

Many commercial rheometer software packages use models which are unsuitable for yield stress fluids and employ a Newtonian analysis for materials which are clearly non-Newtonian. A competent testing facility will be aware of this and analyse the raw instrument readings appropriately rather than relying on inappropriate automated analyses.

\subsection{Rheological models}

Flow data, or shear stress $(\tau)$ as a function of shear rate $(\gamma)$ is often presented in the form of a rheological model. The most commonly used models are the Bingham and Herschel-Bulkley models, shown in Equations 1 and 2 respectively.

Bingham Model:

$$
\tau=\tau_{y B}+\eta_{B} \dot{\gamma}
$$

Herschel-Bulkley Model:

$$
\tau=\tau_{y H B}+K \dot{\gamma}^{n}
$$


The Bingham Model has historically been used for prediction of pipeline transport requirements, where the shear rates of interest are of the order of 10 to $100 \mathrm{~s}^{-1}$. For pipeline start-up conditions, rake drive requirements and vessel agitation, the shear rates of interest are much lower (zero for pipeline start-up conditions and rake drive requirements and 5 to $15 \mathrm{~s}^{-1}$ for agitation), so the Bingham parameters are largely unsuitable.

Although fluid models are convenient the constants $\tau_{\mathrm{yB}}, \tau_{\mathrm{yHB}}, \eta_{\mathrm{B}}, \mathrm{K}$ and $\mathrm{n}$ are fitting parameters only, and may not be representative of the true flow characteristics of the material. This is particularly relevant in the case where a highly shear thinning material is described using a Bingham model. At high solids loadings, few slurries/pastes display true Bingham behaviour, yet Bingham parameters are often quoted. The Bingham model extrapolates high shear rate data to zero shear rate and may result in a significant overestimation of the true yield stress (the stress required for flow to occur) while the constant Bingham viscosity $\left(\eta_{\mathrm{B}}\right)$ does not indicate the variation in the true viscosity, defined as the ratio of the shear stress to the shear rate at a particular shear rate, especially at low shear rates. Figure 7 shows the discrepancy between the true, vane yield stress (shown in brackets) and the Bingham yield stress $\left(\tau_{\mathrm{yB}}\right)$ for a cemented lead/zinc tailings paste. Clearly, designing based on the Bingham yield stress would result in excessive conservatism, especially at high solids concentrations, where the Bingham yield stress may indicate unfeasible or uneconomic operation. The Herschel-Bulkley model generally provides a far better representation of the actual material behaviour.

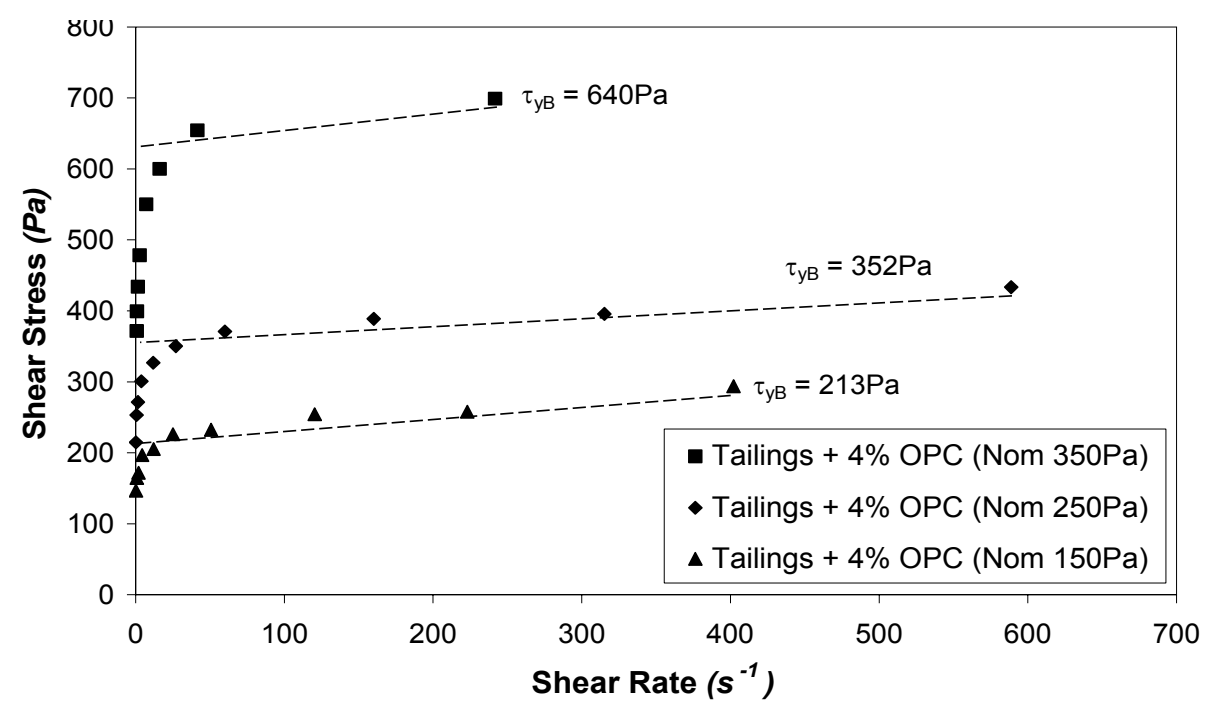

Figure $7 \quad$ Flow curves for a cemented lead/zinc tailings paste

\section{CONCLUSIONS}

A simplistic roadmap has been developed to assist designers and operators to navigate through the following; rheological questions to ask, issues to address - from sampling to testing protocols, data analysis and informed data application. Figure 8 attempts to simplify this complex process by considering the effect of ore 
mineralogy, sample preparation and management, baseline tests required and test methods and data analysis and application.

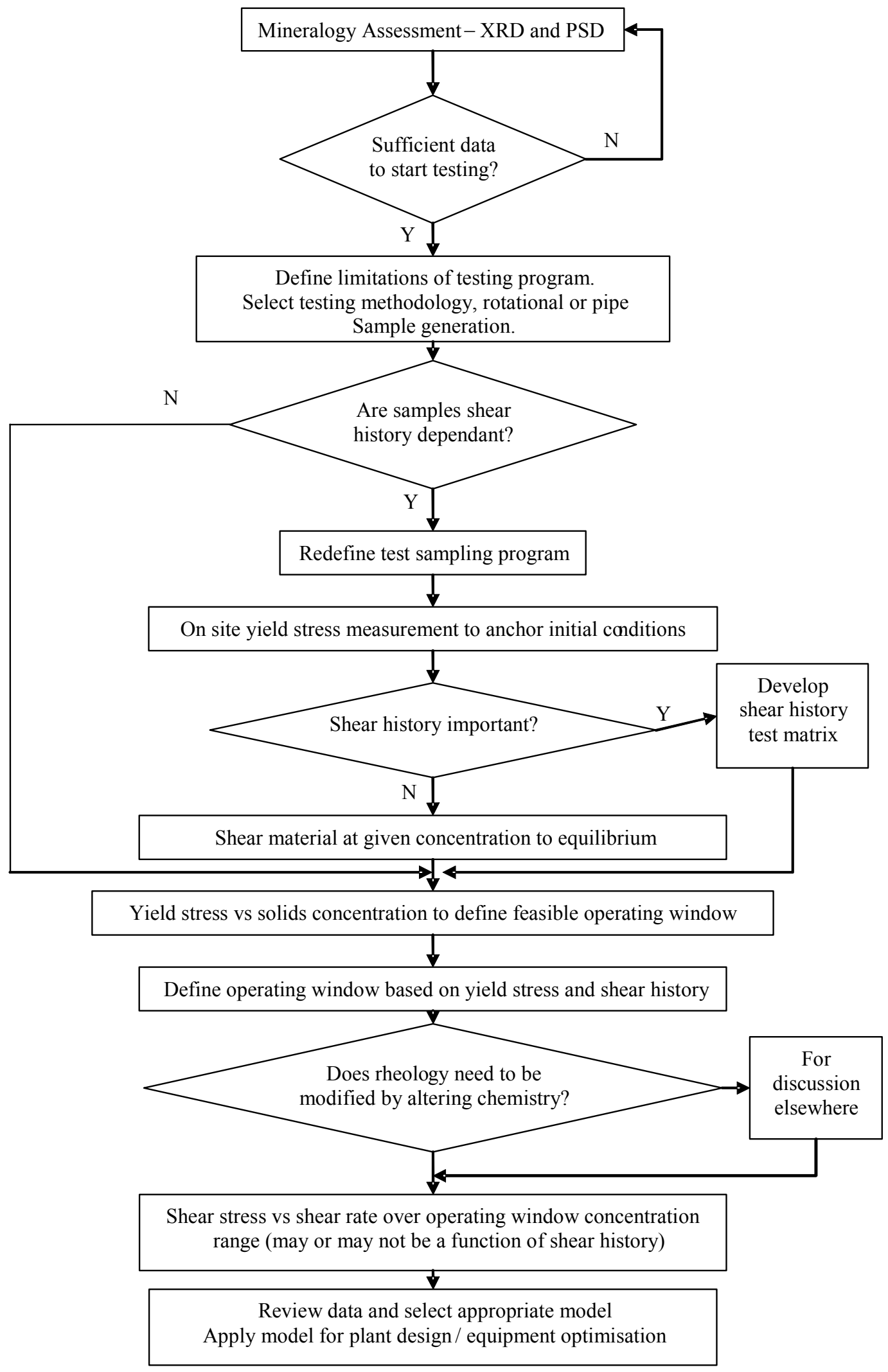

Figure 8 Preliminary 'rheology roadmap' 